Voix et Images

voixetimages

\title{
La Revue d'histoire littéraire du Québec et du Canada français
}

\section{René Dionne}

Volume 12, numéro 2 (35), hiver 1987

Jacques Brault

URI : https://id.erudit.org/iderudit/200637ar

DOI : https://doi.org/10.7202/200637ar

Aller au sommaire du numéro

\section{Éditeur(s)}

Université du Québec à Montréal

\section{ISSN}

0318-9201 (imprimé)

1705-933X (numérique)

Découvrir la revue

\section{Citer cet article}

Dionne, R. (1987). La Revue d'histoire littéraire du Québec et du Canada français. Voix et Images, 12(2), 287-294. https://doi.org/10.7202/200637ar d'utilisation que vous pouvez consulter en ligne.

https://apropos.erudit.org/fr/usagers/politique-dutilisation/ 
ÉTUDES 287

\title{
La Revue d'histoire littéraire du Québec et du Canada français
}

\author{
par René Dionne, Université d'Ottawa
}

La Revue d'histoire littéraire du Québec et du Canada français (RHLQCF) a été créée en 1979 par le Comité de recherche francophone de l'Association des littératures canadiennes et québécoise (ALCQ)'. Après avoir étudié pendant trois ans la situation et les conditions de la recherche dans le domaine littéraire québécois et canadien-français. les huit membres ${ }^{2}$ de ce comité interuniversitaire et interrégional avaient conclu principalement à la nécessité d'éditer critiquement un certain nombre d'œuvres québécoises et canadiennes-françaises ${ }^{3}$ et de créer une revue d'histoire littéraire qui serait à la fois un débouché pour la recherche et un moyen de communication entre chercheurs ainsi qu'un instrument de diffusion de cette recherche à l'extérieur du Canada (particulièrement dans les universités où naissaient des Centres d'études canadiennes et dans celles dont les départements de littérature commençaient à s'intéresser aux 'œuvres canadiennes) ${ }^{+}$. C'est en fonction de ces objectifs qu'ont été créées les sept sections que compte, sauf exception ${ }^{5}$, chaque numéro de la RHLQCF. 


\section{Le contenu des numéros}

\section{1. Études}

La première section regroupe des "Études" dont le thème commun donne au numéro son titre; elles portent tantôt sur une situation générale ou un genre littéraire, tantôt sur des problèmes particuliers, tantôt sur un auteur ou sur une cuvre.

Ainsi le" premier numéro, "Situation de l'édition et de la recherche", constituait une sorte d'inventaire du tıavail qui avait été fait et de celui qu'il importait de faire sur le corpus littéraire du Québec et du Canada français, depuis les récits de Jacques Cartier jusqu’à Bonheur d'occasion; cet inventaire a servi de base à l'élaboration du projet "Corpus d'éditions critiques". Les collaborateurs d" "Aspects et problèmes" (no 2) évoquaient à la fois des problèmes de méthodologie générale et des difficultés propres à l'histoire littéraire du Québec, puis ils traitaient de certains problèmes particuliers, tels ceux que posent la recherche sur les écrits de la Nouvelle-France. l'étude des textes du dix-neuvième siècle, les relations de l'histoire et de la critique. la réception des auvres aujourd'hui; ils proposaient ensuite des voies pour l'étude des poètes artistes (1910-1930), du discours romanesque et du corpus poétique des années quarante et suivantes, voire de la formation littéraire dans l'enseignement classique au Québec de 1852 à 1968.

L'édition savante ayant été l'une des préoccupations majeures du comité qui a donné naissance à la RHLQCF, c'est tout naturellement que trois des douze premiers numéros ont été consacrés à l’édition critique. Le problème fut d'abord considéré de façon générale, mais pratique, dans le numéro 4 ("L'Édition critique»), à travers le rapport que faisaient de leur expérience six éditeurs (Michel Bideaux, John E. Hare, Odette Condemine, Armand et Bernadette Guilmette. Benoît Lacroix) de textes d'auteurs canadiens-français (Jacques Cartier, Joseph Quesnel, Octave Crémazie, Nérée Beauchemin, Jean-Aubert Loranger, Saint-Denys Garneau). Par la suite, deux équipes de chercheurs présentèrent les projets qu'elles avaient en chantier: l'une, dirigée par Jean-Cléo Godin. fit état de ses travaux sur les ceuvres d'Alain Grandbois (no 8); l'autre, sous la direction de Jacques Allard. communiqua les premiers résultats de ses recherches en vue de l'édition des œuvres d'Hubert Aquin (no 10).

Fondée à un moment où les études de littérature régionale prenaient de l'envergure, la RHLQCF a accordé beaucoup d'importance aux travaux des chercheurs non montréalais. «Littérature régionale» (no 3) traitait des littératures de diverses régions du Québec et du Canada français: l'Acadie, l'est du Québec, la Mauricie, les Cantons de l'Est. l'Ontario français. «Frontières" (no 12) est presque tout entier consacré aux littératures des minorités francophones non québécoises de l'Amérique du Nord, celles de l'Acadie. de la Nouvelle-Angleterre, de l'Ontario et de l'Ouest canadien; il laisse apercevoir une partie des richesses que recèlent ces littératures en même temps qu'il met en lumière l'importance de la recherche universitaire en ces régions. 11 contient également une étude sur la québécité de Colas et Colinette ou le Bailli dupé. 
caractère qu'une tradition critique obstinée avait toujours refusé à cette pièce de Joseph Quesnel, et un article qui révèle l'influence du Français Prosper Blanchemain sur la carrière du poète Louis Fréchette.

Pour stimuler la recherche dans des secteurs peu explorés, la RHLQCF a publié quatre numéros dont les études, classées selon l'ord re chronologique de leurs sujets, jalonnent de points de repère ou d'ancrage l'histoire d'un domaine ou d'un genre. "Le Théâtre" (no 5), après des "prolégomènes à une histoire du théâtre» par Laurent Mailhot, contient des articles sur le théâtre politique du dix-neuvième siècle, l'accueil que les Montréalais ont fait jadis à Sarah Bernardt, une pièce de Julien Daoust, le théâtre paroissial, les femmes et le théâtre. le théâtre québécois à Toronto, le théâtre franco-manitobain et le théâtre d'expression française en Acadie; à défaut de constituer une histoire suivie du genre théâtral au Québec et au Canada français, ces essais mettent à la disposition des chercheurs une mine de renseignements qu'utiliseront avec profit les futurs historiens du genre. Il en va de même du numéro sur les "Revues littéraires du Québec» (no 6, préparé par Clément Moisan), qui traite de quatorze revues - depuis les «Soirées canadiennes (1861-1865) jusqu’à Jeu (né en 1976) - et de plusieurs «fanzines» québécois, et du numéro sur «la Littérature personnelle» (no 9, préparé par David M. Hayne et Pierre Hébert), dont l'introduction («Pour une évolution de la littérature personnelle au Québec: l'exemple du journal intime", par Pierre Hébert) est suivie d'études sur le récit d'enfance, les écrits autobiographiques de Jean de Brébeuf. le Journal d'Henriette Dessaulles, les Mémoires intimes de Louis Fréchette, la correspondance d'Olivar Asselin et de Claude-Henri Grignon et sur une querelle épistolaire inédite entre Camille Roy et Jean-Charles Harvey. Le numéro II, "Littéráture québécoise et cinéma " (préparé par Laurent Mailhot et Benoît Melançon), explore un domaine laissé passablement en friche jusqu'ici. C'est la première fois, en effet, que l'on trouve rassemblés un si grand nombre d'articles sur les rapports que la littérature québécoise et le cinéma ont entretenus entre eux, depuis les premières adaptations à l'ONF (1939-1965) jusqu'aux toutes dernières adaptations de romans québécois (Maria Chapdelaine, Bonheur d'occasion, les Plouffe, Kamouraska, etc.) et aux scénarios de Réjean Ducharme (les Bons Débarras et les Beaux Souvenirs), sans oublier "les Indiens de celluloïd». Des numéros du même genre sont en préparation sur la critique littéraire (no 14; sous la responsabilité de David M. Hayne), la littérature radiophonique (no 17; responsable: Renée Legris) et la littérature québécoise au Canada anglais (no 19; responsable: Pierre Hébert).

Des douze premiers numéros, il n'y a que le septième qui ait porté sur un seul auteur. François-Xavier Garneaú en l'occurrence. Le prochain, préparé par Clément Moisan. présentera l'histoire de Menaud, maitre-draveur ; d'autres auront pour titre "Alfred DesRochers et ses correspondants" (no 15; responsable: Richard Giguère), "Ringuet" (no 16; responsable: Guildo Rousseau), "Jacques Ferron" (no 18; responsable: Pierre Cantin). Dans ces numéros, conformément à l'objectif principal de la revue, l'on accordera une importance spéciale à l'histoire des textes et à tout ce qui peut contribuer à leur éventuelle édition critique. 


\section{Notes}

En principe, les textes de la section "Notes" sont des textes brefs qui précisent ou complètent des données d'histoire littéraire. Tel fut bien le cas au début de la revue [ .g.. dans le no 1 , «les Difficultés techniques de l'édition québécoise au début du XIXe siècle: Joseph Quesnel et l'impression de la musique de Colas et Colinette (1808)" ], alors que les sujets abordés dans cette partie n'étaient pas nécessairement reliés au thème de la première section [ $\because$.g., dans le no 5 (Le Théâtre). "Angéline de Montbrun ou les Abîmes de la critique" ]. Peu à peu cependant, les articles thématiques abondant, cette partie' n'a plus inclus que des textes se rapportant au thème du numéro, soit indirectement [ $\mathrm{V}, g$., les deux numéros sur la littérature régionale contiennent des notes sur la langue acadienne], soit directement [ $\mathrm{l}$.g. le numéro sur l'édition critique renferme une note sur l'édition inédite d'un manuscrit franco-ontarien; celui sur l'édition des œuvres d'Aquin, un "Inventaire des fonds" et une "Mise à jour (1983-1984) de la bibliographie analytique d'Hubert Aquin" par Jacinthe Martel].

\section{Documents}

Le titre de la troisième section, "Documents», doit s'entendre idéalement de textes inédits ou difficilement accessibles, dont la publication est de nature à intéresser les historiens littéraires. Ces textes sont régulièrement précédés d'une présentation qui les situe historiquement et en montre l'importance et accompagnés de notes infrapaginales qui les éclairent. Mentionnons, à titre d'exemples, les éditions critiques de textes d'Antoine Gérin-Lajoie (no I) et de Louis Fréchette (no 4) et celle du Journal de voyage de M. Saint-Luc de La Corne, Écuyer, dans le navire l'«Auguste», en l'an 1761 (no 2). D'abord indépendante du thème du numéro, la section «Documents" s'en est rapprochée peu à peu. jusqu'au point de constituer avec les deux premières sections un ensemble imposant.

\section{Livres}

La section «Livres" a pris de lampleur afin de répondre davantage aux besoins et aux désirs - souvent manifestés - des lecteurs de la revue. Au début, elle pouvait se présenter soit comme un article qui faisait connaître la littérature récente sur un sujet (no 1. "Des livres, des idées et des hommes d'ici au XIX ${ }^{\mathrm{e}}$ siècle»), soit comme une suite de comptes rendus (no 2). Cette seconde façon de faire a prévalu. et nous essayons maintenant de rendre compte du plus grand nombre possible d'ouvrages reliés à l'histoire littéraire (éditions savantes, anthologies, monographies, études d'oeuvres, etc.); 47 ouvrages ont été critiqués dans les douze premiers numéros.

\section{Thèses}

Les auteurs de thèses récentes sont invités à présenter leur ouvrage, selon un schéma préétabli (table des matières, résumé, données diverses), dans la section «Thèses", à la condition que le sujet traité intéresse les historiens de la littérature; il peut s'agir de thèses portant sur l'ensemble d'une période ou sur un groupe d'auteurs, sur un écrivain et son œuvre, sur la langue d'une 
période, sur l'histoire des idées, etc. Lä présentation de ces thèses permet de faire connaître rapidement aux chercheurs des travaux qui ne seront pas publiés en livres ou qui le seront dans les délais plus ou moins longs; jusqu'ici. 20 thèses, soit treize de doctorat et sept de maîtrise, ont été présentées.

\section{Bibliographie}

La section bibliographique est née de la constatation - faite par le Comité de recherche francophone de l'ALCQ - que, en l'absence de bibliographies générales ou particulières exhaustives (sur une période, un sujet, un auteur, etc.), les historiens et les professeurs consacraient individuellement beaucoup d'heures à des recherches bibliographiques qui, en plus d'être forcément partielles - les moyens ou le temps faisant défaut à leurs auteurs restaient inconnues de leurs collègues, qui les recommençaient pour leur propre compte.

Ainsi la «Bibliographie de la critique de la littérature québécoise et canadienne-française dans les revues canadiennes" (francophones et anglophones) - à la fois signalétique et partiellement analytique - vise à faire économiser des milliers d'heures d'un temps précieux aux chercheurs et à leur permettre, grâce à une présentation exhaustive de la source critique importante que sont les revues, de fonder plus facilement et mieux leurs études, de pousser davantage leurs recherches et d'exploiter des champs nouveaux ( v.g. histoire de la critique dans certaines revues ou dans un groupe de revues, tableau de la critique dans les revues d'une période donnée, la fortune d'une cuvre ou d'un auteur à travers l'ensemble des revues canadiennes, etc.). Fruit du dépouillement informatisé de quelque 300 revues, la « Bibliographie de la critique.... est publiée par tranches, annuelles d'ordinaire, et constitue près de la moitié du numéro qui l'accueille. Les années 1974 à 1983 ont paru dans les numéros $1,2,3,4,5,6,8$, 10 ; elles occupent 1014 pages et comptent 19557 entrées. L'année 1984 prendra place dans le numéro 14 (été-automne 1987). Va de pair avec cette bibliographie, dont elle compose d'ailleurs la première partie, celle des ouvrages susceptibles d'intéresser les historiens (bibliographies, répertoires, anthologies, histoires, guides, essais, monographies, etc); elle couvre les années 1978 à 1984 dans les numéros $1,2,3,4,6$ et 9 (la liste de 1985 paraîtra dans le numéro 13). À côté du Dictionnaire des đeuvres littéraires du Québec dans lequel les chercheurs peuvent trouver la bibliographie générale des ouvres, la "Bibliographie» de la RHLQCF se présente comme un outil de travail précieux.

Occasionnellement. la section "Bibliographie» est occupée par une bibliographie particulière dont le besoin est ressenti de façon spéciale par les chercheurs. C"est ainsi que nous avons cru bon de publier rapidement (no 7 , p. 79-229; plus de 1200 entrées) une "Bibliographie analytique d'Hubert Aquin, 1947-1982" (sources, œuvres et critiques), par Jacinthe Martel, à un moment où un groupe de chercheurs (l'EDAQ) prépare l'édition critique de l'œuvre aquinienne sur laquelle travaillent également maints chercheurs indépendants. Une très utile filmo-bibliographie par Benoît Melançon sur le cinéma et la littérature au Québec (no 11. p. 167-261) complète d'heureuse façon le numéro intitulé "Littérature québécoise et cinéma". 


\section{Renseignements divers}

La section "Renseignements divers" a pour but de faire connaître aux chercheurs des centres de recherche et de documentation (nos 2, 3, 9), des projets de recherche importants (nos $4,7,8$ ), l'état de ces projets (no 10), la situation de la recherche dans un domaine particulier, des résumés de colloques d'historiens littéraires (nos $1,5,12$ ), etc.

\section{Quelques éléments de politique}

1. Le comité de rédaction choisit les thèmes des numéros en tenant compte des suggestions qui lui parviennent de l'extérieur. Le directeur et un ou deux collègues (membres ou non du comité) prennent alors le numéro en charge. Ils choisissent des sujets qu'ils proposent à des chercheurs qu'ils croient capables de les traiter et intéressés à le faire; ceux-ci donnent leurs réponses (affirmatives dans une proportion d'environ $75 \%$ ); ils suggèrent parfois d'autres collègues et d'autres sujets. Comme les thèmes des numéros sont d'ordinaire annoncés deux ans ou plus à l'avance, un certain nombre d'articles (environ $10 \%$ ) sont offerts sans qu'ils aient été sollicités autrement que par lannonce du thème. Les collaborateurs disposent de douze à quinze mois, parfois plus, pour rédiger et remettre leurs textes. La RHLQCF paraissant deux fois par année, en mai et en octobre, et le manuscrit devant être remis aux Éditions de l'Université d'Ottawa' ${ }^{6}$ quatre mois avant sa parution, les dates de tombée des articles sont le 15 août (pour le numéro du printemps) et le 15 janvier (pour le numéro de l'automne).

2. Les manuscrits sont lus par au moins un membre du comité de rédaction et un ou deux autres lecteurs (membres ou non du comité de rédaction); ils sont jugés et corrigés au double point de vue de leur contenu et de leur forme. Il arrive, mais rarement, qu'un texte soit tout à fait inacceptable; nous le retournons alors à son auteur en indiquant les raisons du refus. Si un texte jugé inacceptable peut être amélioré - ce qui est souvent le cas -, nous demandons à l'auteur (surtout lorsqu'il s'agit d'un texte qui a été sollicité) de le refaire ou de le corriger en tenant compte des remarques des lecteurs; si la seconde rédaction n'est pas davantage acceptable et que la faiblesse du-texte se situe principalement au niveau de la forme, l'un des lecteurs se charge de faire les corrections nécessaires et la version remaniée est soumise à l'auteur qui acceptera ou non qu'elle soit publiée. Plusieurs textes ont paru. qui avaient été refaits ou remaniés jusqu'à trois fois. Ainsi, en sollicitant les textes auprès de personnes que nous jugeons compétèntes et en travaillant avec elles s'il est nécessaire de faire des remaniements et des corrections, nous en arrivons à publier environ $85 \mathrm{p}$. cent des textes sollicités et quelque $50 \mathrm{p}$. cent de ceux qui sont offerts. Nous croyons que cette politique nous permet d'avoir des collaborateurs fidèles et des textes de bonne qualité. Dans la majorité des cas, les textes n'ont besoin que de corrections mineures. de grammaire et de style; ces corrections sont faites par les lecteurs.

3. Le français est la seule langue de la revue, pour le moment du moins. Des traductions peuvent y trouver place, mais à la condition que le texte 
original n'ait pas déjà fait l'objet d'une publication et que l'auteur se charge de trouver et de payer le traducteur. Nous ne publions pas non plus de résumés en anglais des articles, faute d'espace et de moyens financiers.

4. La RHLQCF est d'abord au service des historiens littéraires du Québec et du Canada. En effet, ces derniers ont signé $233(97.9 \%)$ des 2387 textes contenus dans les douze premiers numéros; les cinq autres textes $(2,1 \%)$ ont été rédigés par deux Franco-Américains, un Français et un Australien. Sans exclure les chercheurs étrangers, il s'est trouvé que nous n'avons fait qu'exceptionnellement appel à leurs services; nous nous proposons d'y recourir davantage et espérons que, la RHLQCF étant maintenant connue à l'étranger (plus d'une centaine d'abonnements), des chercheurs de différents pays nous proposeront des textes. La répartition régionale des collaborateurs canadiens vaut la peine d'être notée, car elle marque assez bien le caractère interrégional de la revue et de son comité de rédaction. L'Acadie a fourni dix auteurs $(7.7 \%)$ et quinze textes $(6.1 \%)$; le Québec, 81 auteurs $(62,3 \%)$ et 138 textes $(55,9 \%)$; l'Ontario, 30 auteurs $(23,1 \%)$ et 82 textes $(33,2 \%)$; l'Ouest. cinq auteurs $(3,8 \%)$ et sept textes $(2,8 \%)^{8}$. Fait encourageant, près de $30 \%$ des collaborateurs sont de jeunes chercheurs; ils représentent une bonne part de l'avenir, et c'est sur cette note d'espoir que nous terminons la présentation de la Revue d'histoire littéraire du Québec et du Canada français.

1. Pour les origines de ce comité et les résultats de ses premiers travaux, voir "le Comité de recherche francophone (1976-1978)", dans Situation de l'édition et de la recherche (littérature québécoise ou canadienne-française), travaux du comité de recherche francophone de l'ALCQ, recueillis et présentés par René Dionne, coll. "Documents de travail du Centre de recherche en civilisation canadienne-française", 18. Ottawa, Université d'Ottawa (CRCCF), 1978. p. 9-16; aussi Histoire littéraire du Québec [ tel fut le titre du premier numéro de la RHLQCF], no I (1979): Situation de l'édition et de la recherche, p. 5-103, 259-264.

2. Jacques Allard (UQAM), René Dionne (président, Ottawa), David M. Hayne (Toronto), Laurent Mailhot (Montréal), Marguerite Maillet (Moncton), Réal Ouellet (Laval), Pierre Savard (Ottawa), Antoine Sirois (Sherbrooke). Les travaux de ce comité furent d'abord subventionnés par le Conseil des arts du Canada (1976-1978), puis par le Conseil de recherches en sciences humaines du Canada (1978-1979).

3. En novembre 1978, le Comité confia aux professeurs Roméo Arbour (coordonnateur, Ottawa). Benoît Lacroix (Montréal) - Laurent Mailhot remplaça ce dernier en mai 1980 - et Jean-Louis Major (Ottawa) la réalisation de ce qui est devenu le projet "Corpus d'éditions critiques", subventionné par le Conseil de recherches en sciences humaines du Canada. Pour de plus amples renseignements, voir René Dionne, «Projet ' ALCQ-ECQCF. Éditions critiques de textes québécois et canadiens-français». Lettres québécoises, no 14, avril-mai 1979, p. 43-45; aussi Roméo Arbour, "Le Projet "Corpus d'éditions critiques"», Corpus, no 1, printemps 1982, p. 1-3; Jean-Louis Major, “Un corpus en devenirm, ibid.. p. 4-5.

4. Le 13 octobre 1978, le Comité remit le projet de revue à un comité de rédaction composé de René Diónne (directeur. Ottawa), Richard Giguère (Sherbrooke), David M. Hayne (Toronto). Laurent Mailhot (Montréal) et Clément Moisan (Laval); ce comité s'est adjoint Renée Legris (UQAM) et Marguerite Maillet (Moncton) en 1981 et Annette Saint-Pierre (Collège universitaire de Saint-Boniface) en 1985. 
5. L'abondance de la matière et, conséquemment, le manque d'espace ont amené la suppression des sections 2, 3,4,5.7 dans le numéro 6 ("Revues littéraires du Québec») et de la section 6 du numéro 12 ("Frontières"). Signalons aussi que le manque d'espace nous a forcés de publier à la fin de la première section du numéro 13 deux textes que nous avions acceptés pour le numéro 12 ; nous avions prévu que nous pourrions placer à la fin de la première section des études qui ne seraient pas rellées au thème du numéro, mais dont la publication s'imposerait.

6. Les quatre premiers numéros ont été publiẻs par les Éditions Bellarmin [8100, boulevard Saint-Laurent, Montréal (Québec), H2P 2L9]; les suivants, par les Éditions de rUniversité d'Ottawa [603, rue Cumberland, Ottawa (Ontario), KiN 6N5].

7. Ce nombre ne comprend pas les avant-propos ni les notes préliminaires.

8. L'on notera que, dans ce dernier calcul, en attribuant à chaque coauteur la paternité individuelle de son texte. nous avons compté 247 textes et non 238 comme nous lavions fait au début du paragraphe. 